\title{
Fracaso y punción múltiple en la colocación de catéteres para hemodiálisis en un hospital escuela
}

\author{
Héctor Parra-Lomeli ${ }^{1 *}$, Carlos Jiménez-Bernardino², Ana P. Rico-Portillo ${ }^{1}$, Iris Cazares-Campos ${ }^{1}$, \\ Manuel Guerrero-Ruiz y Sergio E. Prieto-Miranda ${ }^{2}$
}

${ }^{1}$ Departamento de Nefrología; ${ }^{2}$ Departamento de Educación e Investigación. Hospital General Regional N. ${ }^{46}$, Instituto Mexicano del Seguro Social (IMSS), Guadalajara, Jalisco, México

\section{Resumen}

Objetivo: Determinar los factores que influyen en el fracaso y en la realización de múltiples punciones para la colocación de un catéter venoso central para hemodiálisis (CVCHD). Métodos: Estudio descriptivo, analítico, en el que se incluyó a pacientes con requerimientos de hemodiálisis de febrero a octubre del 2014. Las enfermeras de la clínica de accesos vasculares registraron las características clínicas, así como el número de punciones necesarias, o fracaso, para colocar el CVCHD, y sus complicaciones inmediatas. El análisis de los datos se hizo con estadística descriptiva e inferencial según correspondió; se realizó regresión logística multivariada. Resultados: Se analizaron 180 procedimientos, en los cuales se logró colocar 170 catéteres para hemodiálisis (94.4\%). El fracaso en la colocación del CVCHD fue más frecuente en los pacientes con antecedente de catéteres previos (OR: 1.46; IC 95\%: 1.03-2.05). La obesidad fue el único factor que influyó en la necesidad de dar más de tres punciones (OR: 6.3; IC 95\%: 1.5-26). En el 16.7\% de los procedimientos se presentó alguna complicación. Conclusiones: Se observó un aumento del riesgo para fracaso en la colocación de CVCHD con el antecedente de catéteres previos, y la obesidad se asoció a la necesidad de dar más de tres punciones.

Palabras clave: Hemodiálisis. Catéteres para hemodiálisis. Insuficiencia renal. Falla renal. Acceso vascular. Terapia de sustitución renal.

\section{Failure and multiple puncture in the placement of catheters for hemodialysis in a school hospital}

\section{Abstract}

Objective: To determine the incidence of influent factors in failure and multiple punctures for the placement of central venous catheter for hemodialysis (CVCHD). Methods: A descriptive analytical study, patients with hemodialysis requirements from February to October 2014 were included. Nurses of the vascular access clinic recorder clinical characteristics, as well as the number of necessary punctures, fail to place the CVCHD and its immediate complications. The analysis of the data was done with descriptive and inferential statistics as corresponded; multiple logistic regression was done. Results: 180 procedures were analyzed, of which 170 hemodialysis catheters (94.4\%) were able to be placed. The failure of the CVCHD placement was more frequent in patients with a previous catheter history with an OR $1.46(95 \% \mathrm{Cl} 1.03-2.05)$. Obesity was the only

\section{Correspondencia:}

*Héctor Parra Lomelí

E-mail: drhectorparra@ hotmail.com licencia CC BY-NC-ND (http://creativecommons.org/licenses/by-nc-nd/4.0/).
Fecha de recepción: 17-07-2018

Fecha de aceptación: 13-05-2020

DOI: 10.24875/NEFRO.20000046
Disponible en internet: 17-12-2020

Nefro Latinoam. 2020;17:75-80 www.nefrologialatinoamericana.com 
Nefro Latinoam. 2020;17

factor that influenced the need to give> 3 punctures OR 6.3 (95\% Cl 1.5-26). In 16.7\% of the procedures, a complication occurred. Conclusions: There was an increased risk of failure with a history of previous catheters and the obesity was associated with the need to give $>3$ punctures.

Key words: Hemodialysis. Hemodialysis catheters. Renal insufficiency. Renal failure. Vascular access. Renal replacement therapy.

\section{Introducción}

La cateterización de una vena central es un procedimiento extremadamente común. En EE.UU. se estima que se realizan 5 millones de cateterizaciones al año y en el Reino Unido 200,000 al año'. La técnica más utilizada para canalizar vía percutánea las venas centrales es la técnica de Seldinger, en la cual se punciona el acceso venoso y posteriormente se pasa una guía para deslizar el catéter a su localización final.

Actualmente existen lineamentos y recomendaciones que enfatizan la necesidad de evitar el uso de catéter venoso central para hemodiálisis (CVCHD) de forma prolongada por sus complicaciones a largo plazo, como trombosis ${ }^{2}$, estenosis de vasos centrales ${ }^{3}$ e infecciones ${ }^{4}$. A pesar de esto, continúa siendo una práctica usual en los pacientes incidentes con necesidad de tratamiento de sustitución renal ${ }^{5}$.

Los sitios preferidos para la colocación del CVCHD son la yugular interna y las venas femorales, y se trata de evitar la utilización de las venas subclavias por la mayor probabilidad de desarrollar estenosis central ${ }^{6}$. Por ello, las guías internacionales marcan la vena yugular interna (VYI) derecha como la primera opción ante la necesidad de sustitución renal mediante hemodiálisis ${ }^{7}$. Esta ruta es segura y efectiva en manos expertas, con un éxito del $85-95 \%$.

Las complicaciones al colocar un CVCHD ocurren en alrededor del $15 \%{ }^{9}$ de los casos. Algunos factores que se han relacionado negativamente con el procedimiento son el calibre disminuido de la VYI y la obesidad $^{10,11}$.

El ultrasonido como guía para la cateterización de la VYI ha mostrado superioridad contra la técnica a ciegas utilizando marcas anatómicas, y en la literatura se reporta un éxito del 88.1 al 100\%, con una disminución de punciones fallidas del $86 \%$, una reducción de complicaciones y un menor tiempo de procedimiento ${ }^{12-14}$. El grupo NICE (National Institute for Clinical Excellence) recomiemda el uso de ultrasonografía (USG) como método guía de elección para canalizar un acceso central, tanto en niños como en adultos ${ }^{15}$. Sin embargo, se debe considerar el costo económico del equipo y la experiencia del operador ${ }^{16}$.
La colocación del CVCHD es una competencia tanto del nefrólogo como del residente de nefrología, ya que el ultrasonido es una herramienta que no siempre está al alcance, sobre todo en los países en vías de desarrollo. El conocimiento de la técnica guiada por marcas anatómicas debe ser dominado por el especialista para minimizar las complicaciones ${ }^{17}$. En nuestro medio, desconocemos los factores que influyen en los desenlaces negativos durante la colocación del CVCHD.

\section{Material y métodos}

Es un estudio descriptivo-transversal realizado en el periodo del 1de febrero del 2014 al 31 de octubre del 2014 en el hospital formador de residentes de Nefrología, número 46 del Instituto Mexicano del Seguro Social, en Guadalajara, Jalisco, México. Se incluyó a 180 pacientes hospitalizados que requirieron colocación de catéter para hemodiálisis.

\section{Criterios de selección}

Se incluyó a todos los pacientes mayores de 15 años, de cualquier género, con diagnóstico de ERC terminal sin importar la etiología, o bien LRA con criterios de hemodiálisis, determinados por el nefrólogo. También se incluyó a los pacientes prevalentes en tratamiento de hemodiálisis crónica con disfunción o infección del acceso vascular que requirieron un nuevo catéter en otro vaso sanguíneo diferente y cambio de modalidad de diálisis peritoneal a hemodiálisis.

Fueron excluidos los pacientes que requerían recambios de catéter en el mismo vaso sanguíneo, y se eliminaron las hojas de recolección de datos incompletas o mal llenadas.

\section{Procedimientos}

Se acudió al departamento de nefrología y medicina interna, ya sea en el área de hospitalización, urgencias o en consulta externa. El residente de nefrología fue el responsable de la colocación del CVCHD, tanto tunelizados como no tunelizados. Todos los catéteres fueron 
colocados guiados por referencias anatómicas. Se consideró fracaso en la colocación del CVCHD a la imposibilidad del operador de colocar el acceso vascular en determinado acceso venoso y la punción múltiple (PM), como la necesidad de realizar más de tres punciones.

Las enfermeras del departamento de accesos vasculares llenaron las hojas de recolección con los datos obtenidos en el expediente médico a través del interrogatorio al paciente y de observación directa sobre el responsable de colocar el catéter. En la misma hoja se anotó el género, peso y talla, comorbilidades, antecedentes de catéteres previos, causa de la ERC o de la LRA, motivo de la colocación, presencia de edema, nivel de conciencia, presión arterial, últimos exámenes de laboratorio, si fue exitosa la colocación, el número necesario de punciones para colocar el catéter, el vaso sanguíneo puncionado y el año de residencia del operador, o bien si fue un médico adscrito quien lo colocó.

La población total se dividió en pacientes en quienes fracasó y en quienes se logró colocar el CVCHD en el vaso sanguíneo primariamente elegido, así como en los que se requirió tres punciones o menos y en los que se requirió más de tres punciones para su colocación. Para determinar la efectividad del procedimiento se calculó el porcentaje de éxito según el número de punciones necesarias para colocar el CVCHD. Se registraron también las complicaciones inmediatas durante el procedimiento y posterior al mismo mediante radiografía de tórax.

\section{Análisis estadístico}

Toda la información fue vaciada en una hoja de datos de Excel versión 2015 para su análisis. Se utilizó estadística descriptiva, con frecuencias, porcentajes, media y mediana. Se utilizó la U de Mann Whitney y la T de Student para comparar las variables cuantitativas según las características de su distribución. Se utilizó Chi cuadrado para la comparación de variables categóricas. Se realizó un análisis multivariado ajustado por edad, índice de masa corporal, comorbilidades, estado de conciencia, presencia de edema, número de catéteres para hemodiálisis previos y sitio de colocación. Se determinó asociación mediante OR. Para el análisis de la información se utilizó el programa estadístico SPSS versión 22.0. Se calcularon los intervalos de confianza (IC) al 95\% y se consideró significancia estadística una $p<0.05$.

\section{Resultados}

Durante el periodo de estudio se registraron 182 procedimientos de colocación del CVCHD. Se revisaron y

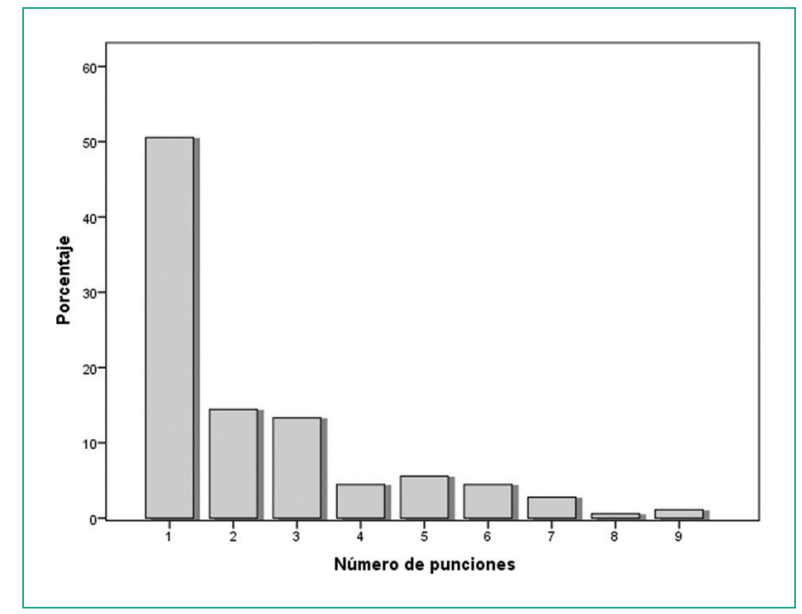

Figura 1. Porcentaje de éxito en la colocación de catéter para hemodiálisis según el número de punciones.

se incluyeron 180 procedimientos y se excluyeron dos pacientes del estudio: uno por registro duplicado y otro por presentar deficiencias en el llenado del instrumento de recolección.

El $64.4 \%$ de los pacientes correspondieron al género masculino, dentro de las variables demográficas incluidas se encontró que el promedio de edad fue de $49 \pm$ 19 años, peso de $72.5 \pm 16.9 \mathrm{~kg}$, talla de $165 \pm 10 \mathrm{~cm}$ e IMC de $26.7 \pm 5.6 \mathrm{~kg} / \mathrm{m}^{2}$. Del total de los pacientes, 166 tenían como diagnóstico ERC: el 38\% (69) secundaria a diabetes mellitus (DM), el 13\% (24) secundaria a hipertensión arterial sistémica (HAS), el 3\% (5) tuvieron como etiología una glomerulonefritis primaria, en el $40 \%$ (61) no se determinó su etiología y el $4 \%$ (7) se atribuyó a diferentes causas.

En 170 de los 180 pacientes (94.4\%) se logró colocar el CVCHD, independientemente del número de punciones o los vasos sanguíneos abordados, el $84 \%$ correspondió a catéteres no tunelizados. El vaso sanguíneo más puncionado fue el yugular interno desde su abordaje posterior $(74 \%)$, seguido del femoral (13\%) y el yugular interno con abordaje medio (5\%). El lado corporal más utilizado fue el derecho, con el $74.5 \%$ de los procedimientos.

Al realizar el análisis de éxito por número de intentos, se encontró que el acceso venoso se colocó en el $50.6 \%$ (91) en la primera punción, en el $14.4 \%$ (26) se requirieron dos punciones y en el $13.3 \%$ (24) se requirieron tres punciones (Fig. 1), con un porcentaje acumulado del $65 \%$ para dos punciones y del $78 \%$ para tres punciones.

Como objetivo primario se analizaron los factores que influyen en el fracaso en la colocación del CVCHD, 
Tabla 1. Fracaso en la colocación de catéter venoso central para hemodiálisis

\begin{tabular}{|c|c|c|c|c|}
\hline Variable & Valor de p & OR & \multicolumn{2}{|c|}{ IC 95\% } \\
\hline Edad & 0.75 & 1.00 & 0.96 & 1.05 \\
\hline Género & 0.66 & 1.34 & 0.36 & 4.96 \\
\hline DM & 0.75 & 1.31 & 0.24 & 7.21 \\
\hline HAS & 0.47 & 0.56 & 0.12 & 2.64 \\
\hline DP & 0.78 & 0.82 & 0.20 & 3.34 \\
\hline LRA & 0.39 & 0.31 & 0.02 & 4.52 \\
\hline N. ${ }^{\circ}$ de catéteres previos & $0.01^{*}$ & 1.38 & 1.06 & 1.79 \\
\hline PAM & 0.17 & 1.03 & 0.98 & 1.08 \\
\hline Paciente inconsciente & 0.99 & 0.99 & 0.07 & 13.53 \\
\hline Complicaciones $^{\dagger}$ & $<0.001^{*}$ & 18.44 & 4.44 & 76.61 \\
\hline Obesidad & 0.55 & 1.53 & 0.38 & 6.20 \\
\hline Sobrehidratación & 0.57 & 0.69 & 0.19 & 2.46 \\
\hline $\begin{array}{l}\text { Análisis multivariado de los facto } \\
\text { clocación de catéter venoso ce } \\
\mathrm{p}<0.05 \text {. } \\
\text { Complicaciones: hematoma, pun } \\
\text { raqueal. } \\
\text { DM: diabetes mellitus; DP: diálisi } \\
\text { C: intervalos de confianza; LRA: }\end{array}$ & $\begin{array}{l}\text { línicos en rel } \\
\text { para hemodiá } \\
\text { arterial, arritm } \\
\text { itoneal; HAS: } \\
\text { n renal aguda }\end{array}$ & hipote & $\begin{array}{l}\text { n y pur } \\
\text { terial s } \\
\text { arteria }\end{array}$ & $\begin{array}{l}\text { ón } \\
\text { émica; } \\
\text { edia. }\end{array}$ \\
\hline
\end{tabular}

la cual se presentó en 28 pacientes (15.5\%). Al realizar el análisis multivariado ajustado para la edad, género, antecedentes de DM, HAS, diálisis peritoneal, LRA, catéteres previos, presión arterial media (PAM) en el procedimiento, estado de conciencia y complicaciones, se encontró que el número de catéteres previos es un factor de riesgo independiente para la falla (OR: 1.46; IC 95\%: 1.03-2.05). En aquellos pacientes que presentaron fracaso aumentó el riesgo para complicaciones $(p \leq 0.001)$ (Tabla 1).

Al realizar un análisis multivariado de los pacientes que requirieron tres punciones o menos y los que requirieron más de tres punciones para la colocación del CVCHD, ajustado para edad, género, antecedentes de DM, HAS, diálisis peritoneal, LRA, catéteres previos, PAM en el procedimiento, estado de conciencia y complicaciones, solo se encontró la obesidad como factor asociado a realizar más de tres punciones (OR: 6.36; IC 95\%: 1.55-26.03).

Se registraron 30 procedimientos con complicaciones, que corresponden al 16.7\%. En 7 procedimientos se registró más de una complicación. La complicación más frecuente fue la punción arterial, con el 11\% (20/180) de los procedimientos, seguida de hematoma,

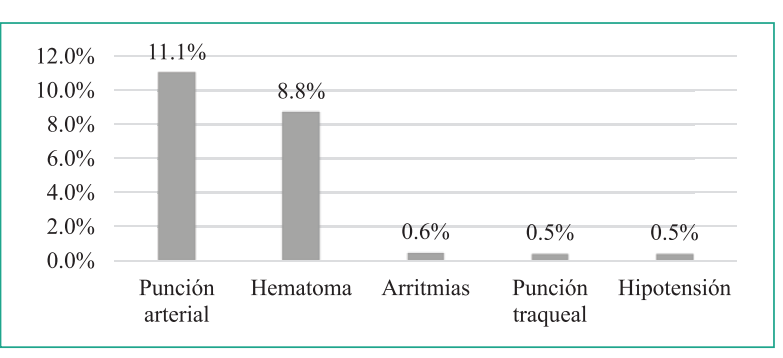

Figura 2. Complicaciones de la colocación del catéter venoso central para hemodiálisis.

y posteriormente se registró un caso de arritmia cardíaca, hipotensión y punción traqueal. No se presentó ninguna defunción asociada a la colocación del acceso vascular, como se muestra en la figura 2.

No se encontró diferencia significativa en el número de complicaciones entre los que requirieron dos punciones y los que requirieron tres punciones para la colocación del CVCHD, con un porcentaje de complicaciones del $4.2 \%$ contra $6.38 \%$, respectivamente $(p=0.45)$.

\section{Discusión}

En este estudio se presentó fracaso en la colocación del CVCHD en los pacientes con antecedente de catéteres previos, por cada catéter que haya tenido el paciente, aumenta de manera significativa la probabilidad de presentar falla en la colocación del acceso venoso (OR: 1.46; IC 95\%: 1.03-2.05). Esta observación no se había hecho con anterioridad. Previamente se habían descrito los factores asociados a fracaso en la colocación del CVCHD, y solo había reconocido a la obesidad como un factor de riesgo cuando el abordaje es yugular anterior ${ }^{11}$.

En el estudio de Macrae, et al. ${ }^{18}$, en pacientes con hemodiálisis se encontró que el número de catéteres previos influía de manera significativa (OR: 2.69; $p=0.004)$ en el diagnóstico de estenosis vascular central por venografía, lo que puede justificar la mayor cantidad de fallas en la colocación del CVCHD encontradas en pacientes con antecedente de catéteres previos.

En la literatura existe poca información sobre los factores que determinan la necesidad de realizar PM para colocar el CVCHD. En nuestro estudio encontramos que los pacientes con obesidad tienen un OR de 6.37 para 
requerir PM en la colocación del acceso venoso, y fue el único factor clínico significativo que encontramos.

Algunos autores han considerado que la obesidad es un factor que impacta en el desenlace de la colocación del acceso venoso dependiendo del abordaje, pero sin describir su relación con $\mathrm{PM}^{11}$. En nuestro estudio encontramos que, en los pacientes con obesidad, la frecuencia de PM fue mayor, sin influir en el porcentaje de fracaso.

Desafortunadamente no existe una definición universal para considerar cuando se han realizado PM en un paciente. Báez, et al. ${ }^{19}$ las describe como una complicación que se presenta hasta en el $15.8 \%$ de los procedimientos, sin embargo, no especifica la cantidad de punciones definitorias del término.

En nuestro estudio se observó que el $78 \%$ de los catéteres se lograron colocar en tres punciones o menos, y las complicaciones fueron similares con los que requirieron una o dos punciones, por lo que estimamos que un punto de corte adecuado para definir este término podrían ser más de tres punciones, ya que este punto ofrece el mayor porcentaje de éxito en la colocación del catéter, sin tener diferencia en la frecuencia de complicaciones.

La colocación del CVCHD guiado por referencias anatómicas tuvo un éxito del $94.4 \%$ en nuestra población estudiada, que concuerda con el rango reportado en la literatura, que va de un 85 a un $95 \%{ }^{8}$. También se observó un éxito en la primera punción del $50 \%$, que es discordante con lo reportado por Augoustides, et al. ${ }^{20}$, donde los residentes de primer año tuvieron una efectividad a la primera punción mayor, del $69.6 \%$. Por otro lado, también se han emitido reportes de efectividad a la primera punción tan baja como del $42 \%{ }^{21}$. El vaso sanguíneo más puncionado fue la vena yugular interna derecha, desde su abordaje posterior, esto a consecuencia de la experiencia de los docentes de canalizar de primera instancia este vaso. Otros estudios muestran la misma tendencia en otros grupos de especialistas $^{19,22}$.

El porcentaje de complicaciones obtenido en nuestro estudio fue similar a lo reportado en la literatura, cuyo rango se encuentra entre el 6.3 y el 16\% $\%^{8,23}$. El hematoma y la punción arterial fueron las complicaciones más frecuentes y solo en cuatro procedimientos el hematoma fue consecuencia de la punción arterial. No hubo casos de neumotórax ni hemotórax, debido a que estas complicaciones son más frecuentes al canalizar las venas subclavias 0 al canalizar la yugular interna desde su abordaje medio ${ }^{24}$.
El presente estudio no controló la técnica, el abordaje ni el año del residente de nefrología que realizó el procedimiento. Nos limitamos a la descripción de antecedentes, factores clínicos que pudieron impactar en el resultado de la colocación del CVCHD. Al ser observado y registrado por un servicio ajeno al nuestro, se minimizó la tendencia del médico a registrar menor cantidad de punciones o de complicaciones del procedimiento.

\section{Conclusión}

Actualmente la colocación del CVCHD guiada por ultrasonido es la técnica de elección en todos los casos siempre que se encuentre disponible. Debido a que se encontró que el antecedente de catéteres previos se asoció al fracaso en la colocación del CVCHD y los pacientes con obesidad presentaron un mayor riesgo de $\mathrm{PM}$, se sugiere que en pacientes con antecedente de CVCHD previos y/u obesidad se evite el uso de la técnica por referencias anatómicas. Con base en el creciente número de complicaciones encontradas posterior a la tercera punción, recomendamos al personal en formación de nuestro hospital realizar tres intentos máximo guiados por referencias anatómicas.

\section{Agradecimientos}

A los compañeros residentes de Nefrología del hospital, así como a las enfermeras de clínica de accesos vasculares: María Guadalupe Ley Ávila, Flor de Belem Jiménez Girón, Laura Verónica Ponce Reséndiz y Erika Luna Ortega, por su apoyo para realizar el estudio.

\section{Conflicto de intereses}

Los autores declaran no tener ningún conflicto de intereses.

\section{Responsabilidades éticas}

Protección de personas y animales. Los autores declaran que para esta investigación no se han realizado experimentos en seres humanos ni en animales.

Confidencialidad de los datos. Los autores declaran que han seguido los protocolos de su centro de trabajo sobre la publicación de datos de pacientes.

Derecho a la privacidad y consentimiento informado. Los autores declaran que en este artículo no aparecen datos de pacientes. 


\section{Bibliografía}

1. Lorchirachoonkul T, Ti LK, Manohara S, Lye ST, Tan SA, Shen L, et al. Anatomical variations of the internal jugular vein: implications for successful cannulation and risk of carotid artery puncture. Singapore Med J. 2012;53(5):325-8.

2. Oliver MJ, Mendelssohn DC, Quinn RR, Richardson EP, Rajan DK, Pugash RA, et al: Catheter patency and function after catheter sheath disruption: a pilot study. Clin J Am Soc Nephrol. 2007;2(6):1201-6.

3. Mac Rae JM, Ahmed A, Johnson N, Levin A, Kiaii M. Central vein stenosis: a common problem in patients on hemodialysis. ASAIO J. 2005; $51: 77-81$

4. Saad TF. Bacteremia associated with tunneled, cuffed hemodialysis catheters. Am J Kidney Dis. 1999;34:1114-24.

5. Clark EG, Akbari A, Hiebert B, Hiremath S, Komenda P, Lok CE, et al. Geographic and facility variation in initial use of non-tunneled catheters for incident maintenance hemodialysis patients. BMC Nephrol. 2016;17:20.

6. Riezebos RK, Schroeder-Tanka J, De Voogt WG. Occlusion of the proximal subclavian vein complicating pacemaker lead implantation. Europace. 2006;8(1):42-3.

7. KDOQI and National Kidney Foundation. KDOQI Clinical practice guidelines and clinical practice recommendations for anemia in chronic kidney disease. Am J Kidney Dis. 2006;47:S11-S145.

8. Jobes DR, Schwartz AJ, Greenhow DE, Stephenson LW, Ellison N Safer jugular vein cannulation: recognition of arterial puncture and preferential use of external jugular route. Anesthesiology. 1983;59(4):353-5.

9. McGee DC, Gould MK. Preventing complications of central venous catheterization. N Engl J Med. 2003;348(12):1123-33.

10. Mey U, Glasmacher A, Hahn C, Gorschlüter M, Ziske C, Mergelsberg M et al. Evaluation of an ultrasound-guided technique for central venous access via the internal jugular vein in 493 patients. Support Care Cancer. 2003;11(3):148-55.

11. Chudhari LS, Karmarkar US, Dixit RT, Sonia K. Comparison of two different approaches for internal jugular vein cannulation in surgical patients. J Postgrad Med. 1998;44:57-62.

12. Troianos CA, Jobes DR, Ellison N. Ultrasound-guided cannulation of the internal jugular vein. A prospective, randomized study. Anesth Analg. 1991;72:823-6.

13. Denys BG, Uretsky BF, Reddy PS. Ultrasound assisted cannulation of the internal jugular vein. A prospective comparison to the external landmark guided technique. Circulation. 1993;87:1557-62.
14. Turker G, Kaya FN, Gurbet A, Aksu H, Erdogan C, Atlas A. Internal jugular vein cannulation: An ultrasound guided technique versus a landmark guided technique. Clinics (SaoPaulo). 2009;64(10):989-92.

15. National Institute for Clinical Excellence. Guidance on the use of ultrasound location devices for placing central venous catheters. Technology Appraisal Guidance No 49, September 2002. https://www.nice.org.uk/ Guidance/TA49

16. Yoshida $H$, Kushikata $T$, Kitayama $H$, Hashomoto $H$, Kimura $F$, Niwa $H$, et al. Time consumption risk of real time ultrasound guided internal jugular vein cannulation in pediatric patients: comparison with two conventional techniques. J Anesth. 2010;24:653-5.

17. McQuillan RF, Clark E, Zahirieh A, Cohen ER, Paparello JJ, Wayne DB et al. Performance of temporary hemodialysis catheter insertion by nephrology fellows and attending nephrologists. Clin J Am Soc Nephrol. 2015:10:1767-72.

18. Macrae JM, Ahmed A, Johnson N, Levin A, Kiaii M. Central vein stenosis: a common problem in patients on hemodialysis. ASAIO J. 2005;51(1):77-81.

19. Báez López Y, Lugo López FE, Rodríguez Ramos N, Báez López JM, Alfonso Pérez RA. Aspectos clínicos relacionados con los catéteres centro venosos temporales y permanentes para hemodiálisis. Revista Cubana de Medicina Militar; 2011;40(2):104-13

20. Augoustides JG, Diaz D, Weiner J, Clarke C, Jobes DR. Current Practice of Internal Jugular Venous Cannulation in a University Anesthesia Department: Influence of Operator Experience on Success of Cannulation and Arterial Injury. J Cardiothorac Vasc Anesth. 2002;16(5):567-71.

21. Sharon Griswold-Theodorson, Hashibul Hannan, Neal Handly, Brian Pugh, John Fojtik, Mark Saks, Richard J. Hamilton et al. Improving patient safety with ultrasonography guidance during internal jugular central venous catheter placement by novice practitioners. Simul Healthc. 2009;4(4):212-6.

22. Pérez Delgado $Y$, Sotolongo Molina $Y$, Muradás Augier M, Vigoa Sánchez L, Lugo López E. Supervivencia y complicaciones de los catéteres para hemodiálisis: nuestra experiencia. Rev Cubana Cir. 2006;45(3-4).

23. Leung J, Duffy M, Finckh A. Real-time ultrasonographically-guided internal jugular vein catheterization in the emergency department increases success rates and reduces complications: a randomized, prospective study. Ann Emerg Med. 2006;48(5):540-7.

24. Vishnu Mahesh Babu B, Kameswara AS, Srikanth B. Comparision of Posterior and Anterior Approaches for Internal Jugular Venous Cannulation - A Prospective \& Randomised Controlled Study. Inter J Sci. Study; 2014;2(2):35-8. 